\title{
MODELING THE MUON COOLING CHANNEL USING MOMENTS*
}

\author{
B. A. Shadwick ${ }^{\dagger}$ and J. S. Wurtele, Department of Physics, UC Berkeley \\ A. M. Sessler, C. M. Celata, and P. B. Lee, Center for Beam Physics, LBNL
}

\begin{abstract}
Using a moment formalism $[1,2]$ we model beam transport in the muon collider cooling channel. This model contains much of the physics we believe to be relevant to muon cooling such as ionization energy loss and multiple scattering. Space-charge forces are currently neglected but can, in principle, be added to the model. Previously, this model has been shown to closely agree with particle tracking [1] while being significantly less computationally intensive. Presently our simulation is limited to the six-dimensional dynamics of the transverse cooling section. A matrix representation of an emittance exchange section is presented. This formulation of emittance exchange can either be ideal (conserving 6-d emittance) or can include energy loss and heating representative of the effects expected in a realistic emittance exchange section. These elements should give our model sufficient generality to enable the preliminary, yet realistic, design of a complete muon cooling channel.
\end{abstract}

\section{INTRODUCTION}

A significant technological challenge in the design of a high-luminosity $\mu^{+} \mu^{-}$collider is the cooling of the muon beam. When the muons are produced the initial beam occupies such a large volume in phase space that efficient acceleration is impractical. The successful reduction of the initial phase-space of the muon beam by a factor of $10^{5}-10^{6}$ is necessary to reach the luminosity goals demanded by highenergy physics applications [3]. Furthermore, this cooling must take place on a time scale that is set by the muon lifetime. Thus, cooling mechanisms such as synchrotron radiation, microwave stochastic cooling and sympathetic beam cooling are all too slow. Of several possible rapid cooling mechanisms, ionization cooling appears to be the most attractive technologically [3]. The basis physics of ionization cooling is well understood $[4,5]$ but detailed simulations are necessary for the design of a realistic cooling channel.

The design process necessarily entails exploration of a large parameter space. The initial stages of the design essentially amount to rejecting unsuitable configurations and identifying promising candidates for further study. Performing this task via particle tracking is laborious. We present a moment description [1,2] of the cooling process that is significant less computationally intensive than full

\footnotetext{
* Supported by the U. S. DoE Division of High Energy Physics under grant No. DEFG-03-95ER-40936 and contract No. DEA-AC0376SF0098

$\dagger$ email: shadwick@beams.lbl.gov
}

tracking but still contains much of the physics relevant to ionization cooling. While this approach is useful in identifying promising designs, particle tracking $[6,7]$ remains an essential step to fully validate and optimize such preliminary designs.

\section{MOMENT MODEL}

Details of a formalism for describing beam dynamics by moments of arbitrary order is given in Ref. [2]. Here, we study transverse cooling considering only moments up to second order. In this model, the centroid is acted upon by the full nonlinear Lorentz forces while the quadratic moments are subjected to forces linearized about the centroid position. For simplicity, we restrict to the case where the beam centroid deviates little from the axis of the channel. ${ }^{1}$ This results in a reduced model where the transverse centroid motion is eliminated and we follow the evolution of $\langle z\rangle,\left\langle p_{z}\right\rangle,\left\langle\delta x^{2}\right\rangle,\left\langle\delta y^{2}\right\rangle,\left\langle\delta z^{2}\right\rangle,\left\langle\delta p_{x}^{2}\right\rangle,\left\langle\delta p_{y}^{2}\right\rangle$, $\left\langle\delta p_{z}^{2}\right\rangle,\left\langle\delta x \delta p_{x}\right\rangle,\left\langle\delta y \delta p_{y}\right\rangle,\langle\delta x \delta y\rangle,\left\langle\delta x \delta p_{y}\right\rangle,\left\langle\delta y \delta p_{x}\right\rangle$, and $\left\langle\delta p_{x} \delta p_{y}\right\rangle$, where $\langle\cdot\rangle$ denotes an average over the beam distribution and $\delta x \equiv x-\langle x\rangle$ etc.

The lowest-order effects of multiple-scattering (MS) are incorporated into the moment equations as

$$
\begin{aligned}
& \left.\frac{d\left\langle\delta p_{x}^{2}\right\rangle}{d t}\right|_{\mathrm{MS}}=\left\langle p_{z}\right\rangle^{2} \frac{d\langle z\rangle}{d t} \frac{d \theta_{0}^{2}}{d s} \\
& \left.\frac{d\left\langle\delta p_{y}^{2}\right\rangle}{d t}\right|_{\mathrm{MS}}=\left\langle p_{z}\right\rangle^{2} \frac{d\langle z\rangle}{d t} \frac{d \theta_{0}^{2}}{d s}
\end{aligned}
$$

where $\theta_{0}$ is the (energy dependent) width of the Molière distribution as given by Lynch and Dahl [8]. Ionization energy loss (EL) enters the single particle equations as a frictional force in the direction of the particle momentum and subsequently enters the moment equations of motion as

$$
\begin{aligned}
\left.\frac{d\left\langle p_{z}\right\rangle}{d t}\right|_{\mathrm{EL}} & =\frac{\left\langle\delta p_{x}^{2}\right\rangle+\left\langle\delta p_{y}^{2}\right\rangle}{2\left\langle p_{z}\right\rangle}\left[\frac{d E^{\prime}}{d s}\left(\left\langle p_{z}\right\rangle\right)-\frac{d E}{d s}\left(\left\langle p_{z}\right\rangle\right)\right] \\
& +\frac{d E}{d s}\left(\left\langle p_{z}\right\rangle\right)+\frac{\left\langle\delta p_{z}^{2}\right\rangle}{2} \frac{d E^{\prime \prime}}{d s}\left(\left\langle p_{z}\right\rangle\right) \\
\left.\frac{d\left\langle\delta p_{x}^{2}\right\rangle}{d t}\right|_{\mathrm{EL}} & =2 \frac{\left\langle\delta p_{x}^{2}\right\rangle}{\left\langle p_{z}\right\rangle} \frac{d E}{d s}\left(\left\langle p_{z}\right\rangle\right) \\
\left.\frac{d\left\langle\delta p_{z}^{2}\right\rangle}{d t}\right|_{\mathrm{EL}} & =2\left\langle\delta p_{z}^{2}\right\rangle \frac{d E^{\prime}}{d s}\left(\left\langle p_{z}\right\rangle\right)
\end{aligned}
$$

\footnotetext{
${ }^{1}$ This assumption is consistent with ICOOL results of simulating the nominal cooling channel [3].
} 

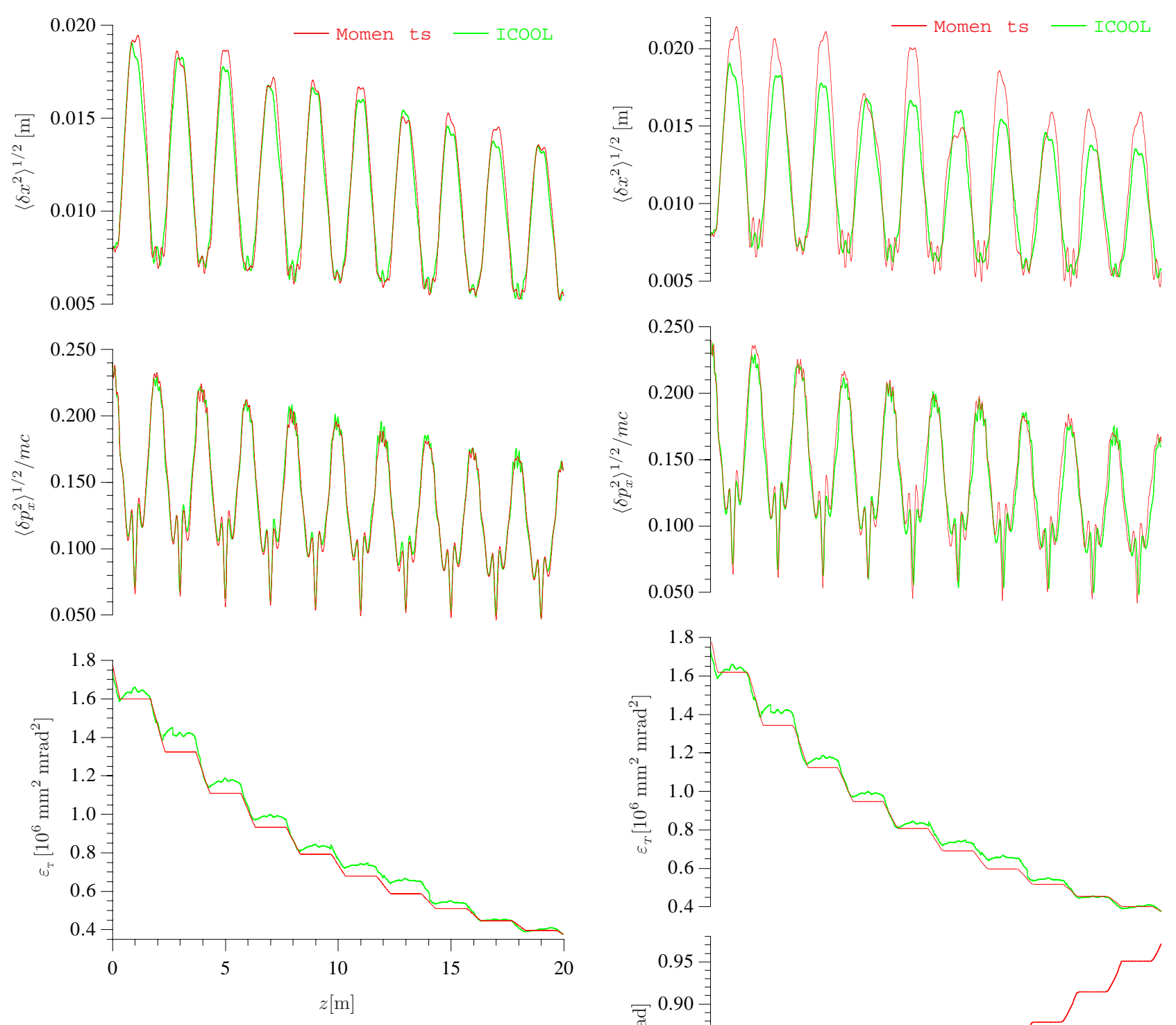

Figure 1: Results of the moment model compared with particle tracking neglecting the longitudinal spread of the beam.

$$
\begin{aligned}
& \left.\frac{d\left\langle\delta p_{x} \delta x\right\rangle}{d t}\right|_{\mathrm{EL}}=\frac{\left\langle\delta p_{x} \delta x\right\rangle}{\left\langle p_{z}\right\rangle} \frac{d E}{d s}\left(\left\langle p_{z}\right\rangle\right) \\
& \left.\frac{d\left\langle\delta p_{x} \delta y\right\rangle}{d t}\right|_{\mathrm{EL}}=\frac{\left\langle\delta p_{x} \delta y\right\rangle}{\left\langle p_{z}\right\rangle} \frac{d E}{d s}\left(\left\langle p_{z}\right\rangle\right) \\
& \left.\frac{d\left\langle\delta p_{z} \delta z\right\rangle}{d t}\right|_{\mathrm{EL}}=\left\langle\delta p_{z} \delta z\right\rangle \frac{d E^{\prime}}{d s}\left(\left\langle p_{z}\right\rangle\right) \\
& \left.\frac{d\left\langle\delta p_{x} \delta p_{y}\right\rangle}{d t}\right|_{\mathrm{EL}}=2 \frac{\left\langle\delta p_{x} \delta p_{y}\right\rangle}{\left\langle p_{z}\right\rangle} \frac{d E}{d s}\left(\left\langle p_{z}\right\rangle\right)
\end{aligned}
$$

where $d E / d s$ is the average energy loss due to ionization as given by the Bethe-Bloch model and equations for $\left\langle\delta p_{y}^{2}\right\rangle$, $\left\langle\delta p_{y} \delta y\right\rangle$, and $\left\langle\delta p_{y} \delta x\right\rangle$ are given by the obvious substitutions. Energy (Landau) straggling has been neglected but its inclusion, in a statistically averaged sense, is planned for the future.

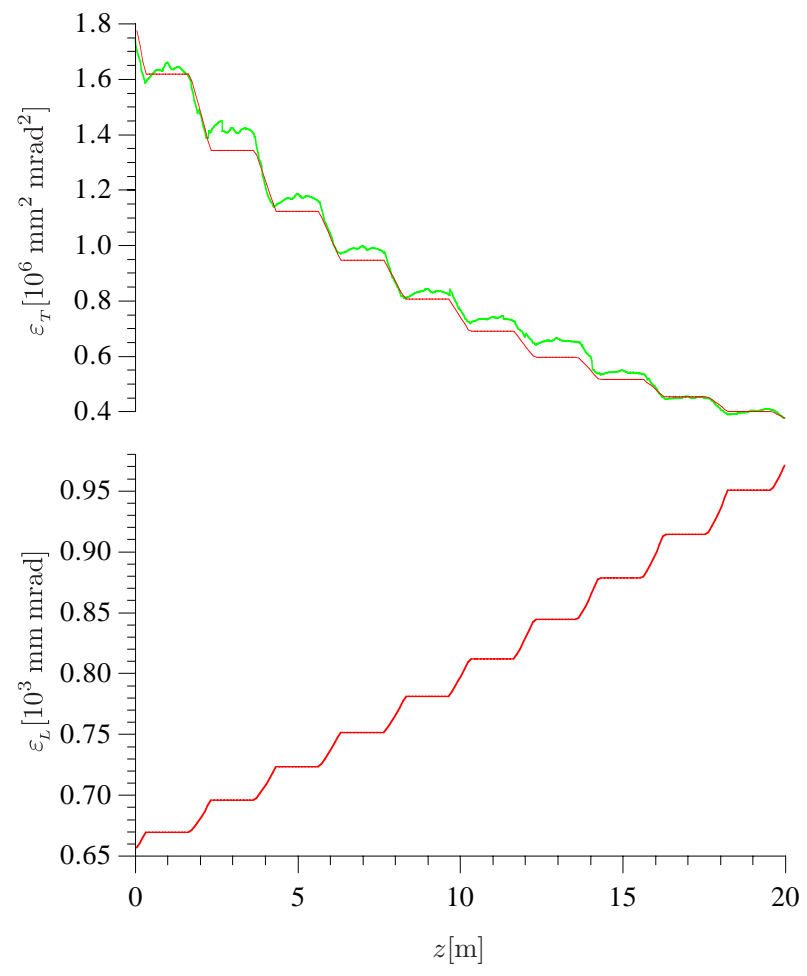

Figure 2: Results of the moment model compared with particle tracking including full longitudinal dynamics.

\section{RESULTS}

We present results from two variants of our model. In the first, shown in Fig. 1, we approximate the initial beam as having no longitudinal extent and use a simple constant gradient model for the linac. Even with this idealization, we obtain excellent agreement with ICOOL tracking results and the only discrepancies can be attributed to dif- 
ferences in the linac models. As can be seen, the normalized transverse emittance (defined as the determinant of the second-order transverse covariance matrix) is constant except where the beam passes through the absorbers. Note even the high-frequency oscillations in $\left\langle\delta p_{x}^{2}\right\rangle$ and $\left\langle\delta x^{2}\right\rangle$ are reproduced by the moment model. In the second variant, shown in Fig. 2, the full longitudinal dynamics, as discussed above, are included and the linac is modeled as a traveling wave structure. Here again good agreement with ICOOL is obtained in the transverse dynamics. The longitudinal agreement is less perfect, with the moment model showing a factor of 1.5 increase in longitudinal emittance compared with the ICOOL prediction of 2.1. Possible sources of this discrepancy are differences in the linac model (most notably an absence of Beryllium windows in the traveling wave linac) as well as incompleteness in our treatment of the material interaction.

\section{EMITTANCE EXCHANGE}

As the beam proceeds through the transverse cooling section, not only is the transverse emittance reduced but the longitudinal emittance is increased due to Landau strangling as well as to the dependence of the ionization energy loss on the beam energy. To prevent the longitudinal emittance from growing without bound it is necessary to periodically exchange emittance between the longitudinal and transverse dimensions. For a preliminary design we feel that it is sufficient to represent this exchange process in a simplified form. One such simplification is shown in Fig. 3. The combination of dispersion and phase rotation has overall effect of converting a beam with large spread in $\delta p_{z}$ and small $\delta x$ into one with a small spread in $\delta p_{z}$ and correspondingly larger $\delta x$.

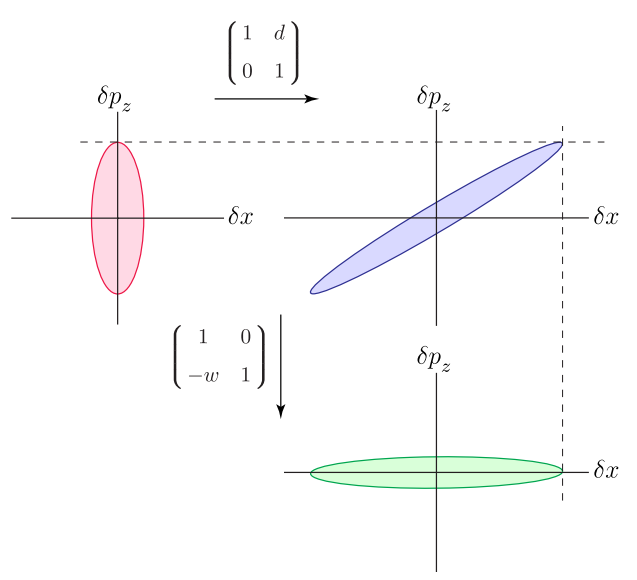

Figure 3: Exchanging emittance between $x$ and $z$.

In terms in the single particle phase space, this action can be expressed as:

$$
W=\left(\begin{array}{cc}
1 & d \\
-w & 1-d w
\end{array}\right)
$$

The second order moments are of interest are $M_{2}=\left\langle\mathfrak{z} \mathfrak{z}^{\mathrm{T}}\right\rangle$, where $\mathfrak{z}^{\mathrm{T}}=\left(\delta x, \delta p_{z}\right)$. The effect on the moments is then
$M_{2} \rightarrow M_{2}^{\prime}=W M_{2} W^{\mathrm{T}}$. Choosing $w$ so that $\left\langle\delta x \delta p_{z}\right\rangle^{\prime}$ is zero leads to ideal exchange:

$$
\begin{aligned}
\left(\begin{array}{cc}
\left\langle\delta x^{2}\right\rangle & 0 \\
0 & \left\langle\delta p_{z}^{2}\right\rangle
\end{array}\right) & \longrightarrow \\
& \left(\begin{array}{cc}
\left\langle\delta x^{2}\right\rangle+d^{2}\left\langle\delta p_{z}^{2}\right\rangle & 0 \\
0 & \frac{\left\langle\delta p_{z}^{2}\right\rangle\left\langle\delta x^{2}\right\rangle}{\left\langle\delta x^{2}\right\rangle+d^{2}\left\langle\delta p_{z}^{2}\right\rangle}
\end{array}\right)
\end{aligned}
$$

A similar matrix can be used to in the $y-p_{z}$ plane completing the transverse-longitudinal exchange. This formulation can be easily extended to reflect the heating and energy loss that occurs in the wedge absorbers of the actual exchange section.

\section{CONCLUSIONS}

We have demonstrated that a moment description of muon cooling contains sufficient physics to yield results equivalent to particle tracking insofar as the average transverse dynamics are concerned. With regard to the longitudinal dynamics the degree of agreement is harder to judge due to the differences in accelerator geometry as well as to physics missing from the moment model. Further work towards understanding the source of the discrepancy with particle tracking results is underway. We are also working to improve the material interactions model in the simulation. The inclusion of an analytic expression for emittance exchange, as discussed above, in the simulation allows for the design a full cooling system.

\section{REFERENCES}

[1] C. M. Celata, A. M. Sessler, P. B. Lee, B. A. Shadwick, and J. S. Wurtele, "A Moment Equation Approach to a Muon Collider Cooling Lattice," in Proceedings of the Sixth European Particle Accelerator Conference, Stockholm (IOP, Bristol, 1998), pp. 1058-1060.

[2] B. A. Shadwick and J. S. Wurtele, "General Moment Model of Beam Transport," paper \#THA130, this proceedings.

[3] C. M. Ankenbrandt et al., "Status of Muon Collider Research," submitted to PRST-AB.

[4] D. Neuffer and A. Van Ginneken, "Simulation Studies of Ionization Cooling," Nucl. Instr. and Meth. A 403, 1 (1998).

[5] A. N. Skrinsky, "Ionization Cooling and the Muon Collider," in Proceedings of the 9th Advanced ICFA Beams Dynamics Workshop. Beam Dynamics and Technology Issues for $\mu^{+} \mu^{-}$ Colliders, Montauk, NY (AIP, NY, 1996), No. 372.

[6] R. Fernow, J. Gallardo, H. Kirk, R. Palmer, J. Norem, D. E. Alwinn, and A. Skrinsky, "Ionization Cooling in the Muon Collider,” Technical Report No. BNL-63615, BNL, 1996.

[7] R. Fernow, J. Gallardo, H. Kirk, and R. Palmer, "Transverse Cooling in the Muon Collider," Technical Report No. BNL65698, BNL, 1998.

[8] G. R. Lynch and O. I. Dahl, "Approximations to Multiple Coulomb Scattering,” Nucl. Inst. and Meth. B58, 6 (1991). 